\title{
EL SISTEMA DE ASEGURAMIENTO DE LA CALIDAD DE LA EDUCACIÓN SUPERIOR CHILENA: LA DEGRADACION ORGANIZACIONAL DE LA INSTITUCIÓN EDUCATIVA
}

\section{THE SYSTEM OF QUALITY ASSURANCE OF CHILEAN HIGHER EDUCATION: ORGANIZATIONAL DEGRADATION OF THE EDUCATIONAL INSTITUTION}

\section{GASTÓN MOLINA DOMINGO*, VALENTINA LETELIER LARRONDO**}

RESUMEN: El presente trabajo se enmarca en el debate en torno al desarrollo del sistema de aseguramiento de la calidad de la educación superior chilena, centrándose en los puntos críticos y el horizonte de posibilidades que presenta, a más de una década de su implementación. A partir de una discusión bibliográfica del tema, se pretende una mínima formalización conceptual de las paradojas que comporta el sistema de aseguramiento de la calidad que, en sus propios términos, podría formularse del siguiente modo: "fortaleciendo" la dimensión de organización de la institución educativa se ha "debilitado" hasta una degradación funcional su dimensión instituyente.

PALABRAS ClAVE: Institución, organización, calidad, acto educativo, deseo.

Aвstract: The present work is part of the debate on the development of the Chilean higher education quality assurance system, focusing on its critical points and the horizon of possibilities the system presents more than a decade after its inception. From a bibliographical discussion of the subject, a minimal conceptual formalization of the paradoxes that the quality assurance system involves is attempted. These, in its own terms, can be formulated as follows: "strengthening" the organizational dimension of the educational institution has "weakened" its instituting dimension to a functional degradation.

KeYwords: Institution, organization, quality, educational event, desire.

Recibido: 2019-07-22. Aceptado: 2020-03-23.

* Doctor en Filosofía. Profesor del Departamento de Filosofía de la Universidad Metropolitana de Ciencias de la Educación, Santiago, Chile. Correo electrónico: gaston.molina@umce.cl. Orcid: https:// orcid.org/0000-0003-2491-3448.

** Magíster en Sociología. Funcionaria de la Universidad de Chile, Santiago, Chile. Correo electrónico: vletelil@uchile.cl. Orcid: https://orcid.org/0000-0002-7371-9791. 


\section{ANTECEDENTES DE CONTEXTO}

- L Sistema de Aseguramiento de la Calidad de la Educación Superior $\mathcal{U}$ (SINACES) en Chile es de reciente data. Sus orígenes se remontan al principio de los años 90, cuando a través del Consejo Nacional de Educación se comienzan a implementar los procesos de licenciamiento, más conocidos como "procesos de acreditación". Estos consistían en la examinación de las nuevas instituciones de educación superior, creadas bajo la Reforma de educación de 1981, cuyas examinadoras eran las universidades públicas o tradicionales. Luego, a fines de los años 90, a través de la Comisión Nacional de Acreditación de Pregrado (CNAP) y de la Comisión Nacional de Acreditación de Postgrado (CONAP), dependientes de la Comisión Nacional de Investigación Científica y Tecnológica (CONICYT), se propone implementar acreditaciones experimentales a los programas de pre y posgrado. Actualmente, los procesos de acreditación para el aseguramiento de la calidad en la educación superior son conducidos por la Comisión Nacional de Acreditación (CNA), creada bajo la ley 20129 en el año 2006.

A cuatro años de la puesta en marcha de dicha comisión, en el año 2010, la CNA acreditó a todas las instituciones de educación superior (IES) que se presentaron ante el organismo: de acuerdo con las actas de sesiones de esta comisión, se acreditaron 16 universidades, 10 institutos profesionales y 7 centros de formación técnica. Esta situación es conocida mediáticamente como el "escándalo de la CNA" y tuvo como resultado que su presidente fuera declarado culpable de cohecho, soborno, lavado de activos y tráfico de influencia, quedando con una condena de 5 años de presidio que actualmente cumple en libertad. Este hecho marca un punto de inflexión en lo que hasta ese momento había sido el mercado de la educación chileno.

Desde la implementación de la ley 20129, que crea el Sistema de Aseguramiento de la Calidad, se han llevado a cabo diversos estudios (Lemaitre y Zapata, 2003; Espinoza y González, 2012; Marshall, 2014; Salazar y Leihy, 2014; Cancino y Schmal, 2014) sobre el funcionamiento y desarrollo de los procesos de acreditación en Chile, que no han dejado de plantear varias críticas, entre las que se destaca justamente la falta de una definición clara del concepto de calidad, pues de ahí derivaría la escasa o nula regulación y fiscalización de los criterios implicados en los procesos de acreditación, así como también la incapacidad que ha tenido el sistema de instalar una cultura permanente de aseguramiento de la calidad. Sin embargo, es preciso adelantar desde ya que esta insuficiencia funciona positivamente. En efecto, 
opera como lo que Laclau (2002) llama un "significante vacío", que precisamente por no estar definido por el contenido de una demanda particular, es capaz de articular una pluralidad de demandas, incluso opuestas y contradictorias, en una formación hegemónica, es decir, en la articulación de un campo discursivo y de una práctica social. Así, "calidad educativa" funciona como un significante vacío que otorga un principio de inteligibilidad, un marco que discrimina entre lo que constituye un problema y lo que resulta evidente. Por ello, dado el impacto que este asunto tiene en los destinos de la política pública sobre la educación superior chilena, nos parece necesario profundizar la investigación sobre los supuestos y las condiciones en que opera el sistema de acreditación en Chile.

Lo cierto es que por lo menos desde el año 2011, la noción de calidad de la educación superior en Chile se ha convertido en el caballo de batalla de todos los sectores políticos. Tal como lo señalan varios autores (Readings, 2012; Ruiz, 2010; Mayol, 2012; Bellei, 2015; Vidal, 2017; Orellana, 2018), dicha noción proviene del mundo empresarial, de donde se toma prestada para ser aplicada al sistema de la educación superior. Entonces, esta lógica funciona como mecanismo de privatización de la educación, una privatización que incluso va más allá de la aplicación de las formas de financiamiento y de regulación empleadas en el mundo empresarial. Como vemos, la coherencia de estos procesos se basa en una inopinada redefinición del problema principal: la educación misma. Es decir, no se trata solamente de la discusión respecto a si es el "sector privado" o el "sector público" -o el armónico equilibrio entre ambos- el que tiene que sostener el esfuerzo por sacar adelante una educación de calidad, como si la finalidad que nombra este término fuera autoevidente y el asunto a discutir solo una cuestión de los medios para lograrlo. ¿Y si el problema fuese más bien dilucidar cuál es el sentido de entender la educación desde la noción de calidad?

\section{INSTITUCIÓN Y ORGANIZACIÓN}

Para abordar este problema de la redefinición de la educación en el marco de los procesos de aseguramiento de la calidad, resulta necesario un breve esclarecimiento de los conceptos de institución y organización. En su artículo "Institución y organización" Yvon Pesqueux (2009) aborda el asunto de la siguiente manera: "La hipótesis general de este texto será la ortogonalidad (y no la oposición) entre 'institución' y 'organización'. Se razonará generalmente a partir de las diferencias y no por la oposición de los dos 
términos" (p. 8). Por su parte, Marilena Chaui, en su libro La ideología de la competencia (2018a), lo mismo que en su artículo "Contra la universidad operacional" (2018b), manifiesta explícitamente un abordaje de estos conceptos en términos oposicionales. Tomando la alusión matemática de Pesqueux podríamos decir que, en principio, ambos autores suponen un plano cartesiano donde disponen los conceptos de "institución" y "organización" al modo de dos vectores en una superficie geométrica euclidiana. En el primer caso la ortogonal establece una lógica diferencial descriptiva, que con una pretensión de objetividad empirista posibilita la distinción de las características de uno y otro vector: universalidad; ideología; público; perspectiva del ser; Institución como constituyente de un derecho (objetivo político); en una dirección. Y generalidad; cultura; privado; perspectiva del crecimiento; Organización como constituyente de una oferta (objetivo económico); en la otra dirección. Ahora bien, la ortogonal supone un punto en común. Este no sería otro que la realidad empírica, tomada como una constatación objetiva y evidente por sí misma, como un dato antropológico al que sólo luego se le superpone la teoría. En este caso, la teoría describe y explica la situación desde dos nociones que serían dos maneras de organizar la compleja trama de la realidad: la institucionalidad política y la organización corporativa.

En el segundo caso, Chaui (2018b) toma una posición. Por ello establece en esa superficie cartesiana los mismos vectores, pero de modo oposicional, siendo el punto en común ya no la realidad empírica sino un encuentro conflictivo:

la universidad pasó a ser afrontada como una organización social ¿Qué significa, entonces, pasar de la condición de institución social a la de organización social? Antes que nada significa pensar una institución a partir de la idea y de la práctica de la administración. (p. 224) (...)

Volcada hacia su propio ombligo, pero sin saber dónde se lo encuentra, la universidad operacional [organización] opera y por eso mismo no actúa [institución] No sorprende, entonces, que ese operar coopere con su continua desmoralización pública y degradación interna. (p. 227)

En términos de información podríamos decir que ambos autores no difieren sustancialmente y nos entregan un valioso conocimiento para el análisis que nos convoca. Lo interesante, sin embargo, es la forma en que lo presentan. La lógica oposicional de Chaui muestra que el dato primero nunca es la realidad empírica en su desnuda constatación. Entre ambos tex- 
tos encontramos una lucha de fuerzas que se despliega como un conflicto discursivo entre las maneras de abordar un mismo asunto: el dato primero aquí es la propia relación diferencial en tanto acción eminentemente sociopolítica.

Tenemos entonces la lógica diferencial de la ortogonal y la lógica conflictiva de los vectores opuestos. Proponemos acá una tercera posibilidad para formalizar el problema de la educación: una lógica paradójica de la "institución" y la "organización" articulada topológicamente. Desde este punto de vista la institución y la organización no son vectores dispuestos en una superficie, sino la misma superficie de una banda cuya torsión interna constituye a los términos, como en una Banda de Moebius. Aquí la organización es la dimensión instituida, el "reverso" de la dimensión instituyente de la institución. En otras palabras, la relación topológica entre institución y organización es la misma que encontramos entre la dimensión ontológica de lo político (la apertura de nuevos horizontes de sentido) y la dimensión óntica de la política (la gestión de los horizontes de sentido ya sedimentados) (Mouffe, 2007), siempre que comprendamos que el antagonismo instituyente de la ontología es la torsión interna que agujerea a lo instituido ónticamente y no un simple o complejo ámbito trascendente que esté más allá de la facticidad. O parafraseando a Alain Badiou (2007a), que a su vez invierte y altera la distinción de Mouffe sustrayendo el problema del sentido, diríamos: la política no ha sido nunca más que la ficción de una trama organizacional de los saberes donde lo político instituye el agujero del acontecimiento que sostiene una verdad. "Educación siempre ha querido decir (salvo en montajes opresivos o pervertidos) sólo esto: disponer los saberes de una forma tal que alguna verdad pueda agujerearlos" (Badiou, 2009, p. 54). En otros términos, la educación nombra desde siempre el acto de emancipación respecto de las opiniones consabidas que se imponen a partir del propio consentimiento.

La paradoja la podemos esbozar así: la "educación de calidad", que en la organización corporativa del "enfoque por competencias" despliega su dimensión instituida, con los mismos procedimientos evaluativos y prospectivos necesarios a los procesos de adquisición de habilidades que pretenden asegurarla, tapona el agujero del acto educativo (de Lajonquiére, 1999), la dimensión instituyente de la educación. El enfoque por competencias, que ha sido la manera de gestionar curricularmente la calidad en la educación, aboga por el desplazamiento de un modelo basado en la transmisión teórica de contenidos rígidos y descontextualizados, a otro basado en la práctica de adquisición de habilidades flexibles a través de un aprendizaje significativo 
que asuma la complejidad de los contextos. El empirismo psicopedagógico se hace aquí un lugar e interviene de forma relevante. Proporciona los patrones comportamentales, medibles y evaluables por medio de evidencias, que posibilitan trazar las líneas de desarrollo que se espera que los estudiantes puedan desenvolver para una mejor adaptación al entorno. Al mismo tiempo, la psicopedagogía dispone el conjunto de estímulos adecuados para potenciar reflexivamente, aprendiendo a aprender, la adquisición de las habilidades y competencias significativas, aquellas que se trazan entre el perfil de ingreso y los aprendizajes esperados, en el horizonte de un mejor servicio a la sociedad.

Ahora bien, si el modelo psicopedagógico, que implementa el enfoque por competencia, que a su vez operacionaliza la noción de calidad, tiene en las categorías de la pasividad y la actividad uno de sus presupuestos más importantes, proponemos que es aquí donde se pone en juego la destitución de la dimensión instituyente de la educación. Dicho de otro modo: en la administración organizacional de los saberes bajo la particular gestión del enfoque por competencia, lo que se pierde es la institución de las verdades. En relación con este punto tomamos de Badiou (2013a) el siguiente aserto: "En una concepción matematizada, la genericidad (o el agujero) de lo Verdadero no implica ni actividad ni pasividad, sino más bien trayectos, y encuentros" (p. 53). La verdad es aquel encuentro contingente que opera un desvío de los saberes. No se entiende aquí las verdades como algo que se pueda llegar a saber, pues las verdades toman cuerpo en esa anomalía que desordena y desestabiliza los saberes. Una verdad acontece como un encuentro contingente que se inscribe anómalamente en los trayectos del saber. Es en este sentido que toda verdad tiene un carácter subjetivo, en la estricta medida que supone una subjetivación. Mientras los saberes se aplican, las verdades suponen una implicación. Los saberes que se cierran al modo del conocimiento empírico y la información son los que se pueden gestionar. Para estos esperamos estándares de calidad organizacional. Pero los saberes dispuestos a ser agujereados por las verdades interrumpen y deslocalizan, sin anularlos, sus trayectos, y por ello el pensamiento se erotiza, y no solo se gestiona. Esta erotización del pensamiento, sin la cual hay instrucción, pero no educación, presupone ese singular encuentro condensado y extensamente trabajado en el concepto psicoanalítico de transferencia (Lacan, 2009a). Si por un lado encontramos la transferencia de un deseo, por otro lado, a la organización corporativa de la educación le corresponde, en el plano de la economía libidinal, el factor marcado por el goce, aquello que precisamente obtura la institución del deseo de verdades. 


\section{LA NOCIÓN DE CALIDAD EN EDUCACIÓN}

Señalamos que la psicopedagogía pone en operación el enfoque por competencias, el que a su vez establece los rituales procedimentales que realizan en la práctica cotidiana la noción de calidad. Ahora bien, el concepto de calidad asociado al sistema de educación superior surge a propósito de varias transformaciones sociales mayores. Por una parte, se encuentra el cambio que sufre el rol de los Estados, esto es, el paso del llamado Estado de bienestar a un Estado subsidiario o Estado evaluador, donde se consolida el pasaje desde un ordenamiento simbólico articulado por decisiones instituyentes, a un desorden de lo simbólico expresado en la legitimación de informes facturados por comités de expertos, como instancia de gobierno. Por otra parte, pero en clara connivencia, la emergencia de lo que hoy llamamos sociedad del conocimiento, donde la calificación educacional de las personas, sus habilidades para procesar y organizar información, es la condición principal para integrarse a una sociedad compleja que requiere de competencias flexibles. En este sentido, uno de los problemas que plantea la noción de calidad surge cuando el sistema educativo la mide expresamente como lo haría una empresa o corporación. Al respecto, Bill Readings (2012) señala que los indicadores cuantitativos de calidad, referidos a la educación, son más bien arbitrarios. La contrapartida a esta posición la podemos encontrar en estudios tales como En busca de la calidad académica (Paradeise y Thoening, 2017): “El mundo de la educación superior y la investigación se encuentra actualmente en un desconcertante periodo de transición que exige la evolución y se asemeja a una revolución" (p. 9). Lo realmente desconcertante aquí, es que la consolidación de un modelo organizacional -muy bien descrito en el libro- sea tenido por algo así como una revolución; un acontecimiento disruptivo pensado como una exigencia evolutiva. Como veremos, esta manera de representarse el modelo no es un error, es parte de su lógica operacional, de su pedagogismo. Es necesario insistir, sin embargo, en que la aparente novedad de estos procesos resulta de la propia lógica liberal, como se puede colegir a partir de Pensar la educación. Desde Friedman a Dewey (Vergara y Martin, 2016).

Es importante considerar que, según diversos autores (Ruiz, 2010; Fernández, 2017; Orellana, 2018), la función principal de la universidad es la "búsqueda de la verdad" y no solo estar al servicio de la sociedad. O bien, la mejor forma en que la escuela y la universidad aportan a la sociedad es que aquellas sirvan a los intereses desinteresados de la verdad. Esto implica, sin embargo, la construcción de un concepto de verdad que esté a la altura 
de lo que la época propone. Es en este sentido que resulta ineludible estar atento al trabajo que Badiou (2007b) viene realizando desde hace algunas décadas: la verdad como excepción inmanente o procedimiento genérico. Es deseable y necesario que la educación sirva a la sociedad, pero antes de ello debe estar al servicio de la verdad, es decir, ajena al interés particular y al interés privado. Hoy, una universidad que solo está al servicio de la sociedad es una corporación que dispone el saber en tanto información, lo que posibilita la adquisición de competencias y habilidades "flexibles" para mejor adaptarse a un mercado en constante cambio, es decir, precario (Fernández et al., 2017).

Sin embargo, esta crítica no se reduce a negar de plano toda evaluación, sino a las formas que esta adquiere al suscribir la noción de calidad o excelencia en su versión empresarial privada como único estándar de medición, o el más relevante a la hora de organizar y planificar políticamente la educación superior. Al respecto, Díaz (2016) señala que: "todo aquello que se mueve en desacuerdo con el estándar - por su carácter creativo o por su distancia crítica- es incontable e incalculable, no medible, irreductible" (p. 209), es decir, hay procesos y dinámicas que la educación propicia cuyas lógicas exceden lo cuantificable y, por lo tanto, no pueden ser evaluadas desde una perspectiva de gestión empresarial. La hegemonía de la noción de calidad en las universidades ha significado también que estas estén subordinadas a un cúmulo de indicadores, indexaciones, rankings, benchmarking, managment, entre otros conceptos importados principalmente del área de la economía y de la gestión privada.

En el mismo sentido la noción de calidad aplicada a la educación dispone a esta como inversión individual. En otras palabras, como plantea Readings (2012), "la educación terciaria es percibida simplemente como otro bien durable, de modo que la capacidad de acceder a ella o la relación calidad-precio se vuelven unas categorías entre otras que influyen la opción individual" (pp. 42-43). Por lo tanto, si la educación es un bien de consumo, como sostuvo el presidente chileno y empresario Sebastián Piñera en 2011, no resulta una extravagancia que la educación deba medirse en términos de calidad y que el individuo se comprenda a sí mismo como gestor del yo, como un inversionista. Sin embargo, como señalan Vergara y Martin, (2016) en la mayor parte de los países desarrollados de la OCDE la educación no es un bien económico, sino un derecho universal proporcionado por el Estado, en todos sus niveles. Aunque aquí el problema es que se trata de un derecho al servicio de un individuo cuyo supuesto antropológico es la satisfacción de necesidades y la adaptación a su contexto significativo. 
El derecho a la educación se convierte en el derecho a elegir qué tipo de trayecto desplegar desde la creatividad e innovación, para, de modo paradójico, adecuarse de mejor manera a lo que hay. A esta disposición Badiou (2006) la llama pedagogismo:

él cree poder reducir lo nuevo a la continuación de lo viejo. Una forma particular de pedagogismo, conforme al espíritu del materialismo democrático, es el amontonamiento de resultados puestos en el mismo plano y según el viejo concepto empírico de resultado, de modo tal que la ausencia de discriminación vuelva ilegible el presente. Se propone así una exposición átona de las ciencias, cuya forma real sólo puede ser, al final, las ganancias que se esperan de ellas (las 'aplicaciones' rentables). (p. 94)

\section{LOS SISTEMAS DE ASEGURAMIENTO DE LA CALIDAD}

Actualmente, casi todos los países adhieren a los procesos de aseguramiento de la calidad, cuyos dispositivos y propósitos son más o menos similares, tendiendo todos hacia una definición amplia, y por lo mismo difusa, que se conoce como "adecuación a los propósitos declarados o idoneidad" (Atria y Lemaitre, 2014, p. 27). A pesar de la transversalidad de su implementación, su concepto central es aún impreciso. Funciona, decíamos, como un significante vacío. Respecto de la ambigüedad que envuelve a la noción de calidad, uno de los esfuerzos más aceptados dentro de la comunidad especializada por dar alineaciones al respecto lo encontramos dentro de la literatura internacional. Se trata de la contribución que realizaron Harvey y Green (1993) al esclarecimiento de la noción de calidad. En su célebre trabajo "Defining Quality", estos autores identificaron al menos cinco formas de entender esta noción: calidad como algo excepcional; como perfección o consistencia; como adecuación a una finalidad; como eficiencia en la utilización de recursos y, por último, calidad como transformación del estudiante. Las distintas formas de entender qué es la calidad propuestas por Harvey y Green (1993) evidencian la falta de consenso sobre la definición de la noción en la educación superior, es decir, sus aproximaciones son diversas y responden también a muy disímiles objetivos. Estas diferentes concepciones oscilan entre polos, es decir, entre visiones muy contrapuestas que ubican también en veredas contradictorias los propósitos de la educación superior. Por ejemplo, en su vertiente más economicista la calidad es entendida como la eficiencia en la utilización de los recursos y, en la otra 
vereda, ubicamos otra corriente del concepto de calidad que se comprende como transformación del estudiante. A primera vista, estas visiones pueden asumirse como posiciones muy distintas. Sin embargo, son dos caras de la misma moneda, puesto que, con matices, finalmente el foco está puesto en la producción de buenos profesionales al servicio de la sociedad, que ha sido reducida a una instancia de satisfacción de necesidades articuladas por las demandas del mercado (Fernández, 2009 y Fernández et al., 2017). Lo que el sistema asegura son las aplicaciones rentables de la administración organizacional del saber, en su dimensión cognitiva (para decirlo en los términos del enfoque por competencias), es decir, del saber reducido a información, y en su dimensión procedimental y actitudinal, el de las habilidades y competencias flexibles para saber-hacer con esa información. Esta disposición comprende al individuo, en los rituales de la práctica cotidiana, como un empresario de sí mismo que ha de gestionar, ante todo, sus capitales afectivos. Las habilidades blandas, la empatía y la resolución de conflictos; la motivación y la actitud positiva para entender la precariedad como un mundo abierto de oportunidades que es responsabilidad de los individuos saber aprovechar, en un horizonte de sentido donde la armonía aparece como un dato antropológico, como una necesidad humana que resulta imperativo satisfacer para la plena autorrealización del individuo.

Hemos señalado que el enfoque por competencias es la instancia de materialización y aseguramiento de la noción de calidad a través de su implementación curricular, y la psicopedagogía constructivista el modelo securitario de estimulación y desarrollo que se ofrece como su garantía. Dentro de la bibliografía nacional chilena podemos encontrar diversos estudios en torno al sistema de aseguramiento de la calidad, cuyos aportes van desde el orden de lo propiamente técnico (Marshall, 2014; Cancino y Schmal, 2014; Espinoza y González, 2012) hasta discusiones que incorporan las dimensiones históricas, políticas e ideológicas (Ruiz, 2010; Orellana, 2018; Bellei, 2015; Mönckeberg, 2007 y 2013) que rodean estos procesos. Las "debilidades" y los consecuentes "desafíos" (conceptos que forman parte de la ideología de las competencias como horizonte sedimentado en el que solo es posible la mejora, la optimización y la perfectibilidad) que se observan en el debate sobre el sistema de aseguramiento de la calidad de la educación superior en Chile son múltiples y de diversa índole. Es por esto que, en un esfuerzo de resumen, consideraremos la siguiente clasificación y la ubicaremos en dos grandes áreas: las "debilidades" y "desafíos" que tienen relación con la concepción del sistema, y aquellas concernientes a la política pública propiamente dicha. 
Respecto de las primeras, y dentro de su misma lógica, Marshall (2014) señala que es necesaria la vinculación entre calidad, acreditación y regulación, dado que hasta ahora el sistema ha funcionado sin esta articulación. Además, la regulación y la fiscalización también han estado ausentes. Siguiendo esta línea, la autora manifiesta la necesidad de alinear los instrumentos de aseguramiento de la calidad y de la acreditación con los incentivos de financiamiento del sistema de educación superior. Desde el punto de vista de la concepción del sistema, Marshall -no atendiendo a que se trata de un significante vacío que en ello encuentra su eficacia- respalda la importancia de definir el concepto: "La necesidad de definiciones sobre calidad ha atravesado el conjunto de entrevistas y llega a ser un requisito básico en el debate de nuevas políticas en esta materia" (p. 2013). Los requerimientos aquí expresados provienen del conjunto de los rectores del Consejo de Rectores de Universidades chilenas (CRUCH), vale decir, universidades estatales y universidades privadas anteriores a 1981. Llama la atención para estos actores que el sistema de aseguramiento de la calidad haya operado sin tener claro su concepto principal. Es decir, se evalúa en base a indicadores cuyo elemento de base, la calidad de la educación, no tiene definición unívoca o al menos estándares básicos. Poco se repara, sin embargo, en que es alrededor de esta casilla vacía desde donde ha sido posible organizar el goce archivístico de estos procesos.

Lo que aquí se plantea, tomando la clasificación que hacen Harvey y Green (1993), es que las instituciones de educación superior (IES) chilenas conciben la calidad meramente como eficiencia en la utilización de recursos, es percibida solo como una instancia de control del uso de los recursos financieros. Esta debilidad está presente tanto en el sistema en su conjunto como en las instituciones particulares que lo componen. Lo que se traduce, para los actores implicados, en que los procesos de acreditación actuales no han logrado instalar una cultura permanente de autoevaluación. Siguiendo a Espinoza y González (2012), esto supone el desafío de desarrollar procesos de mejoramiento continuo en todas las funciones universitarias, es decir, docencia, investigación, gestión y prestación de servicios, con un horizonte de futuro. Esta idea apunta a que la instancia de acreditación no sea solo una especie de performance o puesta en escena, sino más bien una práctica cotidiana en todos los ámbitos de la organización universitaria.

Los autores concuerdan en que la noción suscrita por el Estado chileno es ambigua, toda vez que no se ha reflejado en la sociedad chilena un discernimiento acerca de cuáles son las buenas y cuáles son las malas universidades. Salazar y Leihy (2014) plantean que el sello de calidad que dispone 
actualmente el Estado no ha permitido que la ciudadanía discrimine con claridad "entre universidades serias y proveedores dudosos. Esta dificultad, sin embargo, es precisamente la que cierra y clausura sobre sí misma la lógica de la calidad, legitimada así como horizonte siempre posible, nunca alcanzable, dispuesto como un trayecto a recorrer gradualmente. Esta es otra manera de decir que la organización ha de gestionar su propio fracaso funcional, hacer de este fracaso el dispositivo de su implementación. Esto le permite, en efecto, propugnar la lógica del mejoramiento continuo y los trayectos de perfectibilidad infinita. Estos procesos son sustentados desde la psicopedagogía bajo la rúbrica de la tolerancia a la frustración, a la que el individuo está condenado si no la revierte con la adquisición del saberhacer, del "pensamiento positivo" y la motivación.

\section{INSTITUCIÓN DEL DESEO Y GESTIÓN MOTIVACIONAL}

Desde el psicoanálisis el problema real no es aprender a tolerar la frustración, sino asumir la pérdida de la castración, y con ello la institución del deseo (Lacan, 2009b). ¿Se transmite un saber-hacer o un deseo? Antes de esbozar una respuesta es necesario zanjar la diferencia entre deseo y motivación. Digamos que la motivación se refiere a los intereses particulares de cada individuo en relación con los problemas que le presenta su entorno. Todo se juega en esta comunidad entre cuerpos y lenguajes. El individuo estará, presumiblemente, tanto o más motivado mientras más significativa sea la realidad con la que se enfrenta. De hecho, ya esta manera de presentar el asunto estaría descaminada, pues en esta lógica no se trata de enfrentarse a un problema, sino, para el individuo, de "participar" activamente en el esclarecimiento de su contexto, para poder obtener y producir mayor información, y así leer bien los signos que le permitan integrarse y ocupar un lugar en el juego constante de interpretación y re-significación del mundo y la propia identidad. En suma, en tanto individuos, nos motivamos cuando comprendemos, cuando nos reconocemos como seres finitos inmersos en un mundo complejo y relativo, cuando la enseñanza se despliega como un aprendizaje significativo que, a través de una diversidad de saberes y opiniones, nos abastece de sentido. La motivación, entonces, se produce en la demanda y el consumo satisfactorio de sentido. Desde aquí la educación se comprende y degrada al proceso, organizado corporativamente, de los adecuados estímulos para propiciar el desarrollo personal, conforme a la meta preestablecida de los logros adaptativos y el propio bienestar. 
El deseo, en cambio, en términos psicoanalíticos, tiene una textura completamente distinta (Miller, 2011). Siendo esquemáticos, en rigor el deseo es asunto de un sujeto, no de un individuo. En la medida en que, en tanto individuos, somos capturados por un deseo, devenimos sujetos: el deseo es siempre el deseo del Otro, es decir, deseamos lo que los otros desean, y en ello, deseamos ser deseados y reconocidos. Y si bien el deseo es inmortal, hay fijaciones pulsionales, hay la repetición de los afectos, hay deseos que desean su inhibición, su muerte, su anonimato; renunciar a sí mismos. Si pensamos en los tres registros de Lacan, Imaginario, Simbólico y Real, podríamos decirlo de la siguiente manera: la tolerancia a la frustración nos mantiene fijados a las oscilaciones del deseo imaginario -entre la depresión y la motivación-, es decir, en la demanda del reconocimiento. Solo en el trabajo de asumir la castración aceptando la pérdida constitutiva de la plenitud, reconoceremos también que ningún objeto cubre del todo aquella falta constituyente (deseo Simbólico), en la precipitación de un acto, un encuentro, que nos comprometa con lo imposible (deseo Real). Ahora bien, si los tres registros se presentan a la manera de un "nudo borromeo", entonces no se trata de etapas de desarrollo (Schejtman, 2013). El deseo es no-educable: se trata de un impasse en el núcleo íntimo del proceso educativo, que exige que la gradualidad de este proceso se precipite en un acto educativo.

La diferencia entre motivación y deseo la podemos pensar también desde la distinción entre uso privado y uso público de la razón (Kant, 2004). El "uso privado de la razón", es decir, el que el individuo ejerce asumiendo la ley del mundo, las reglas del juego de lenguaje en el que está inserto, supone el interés individual de la motivación particular-general por la correcta aplicación de los saberes, en busca del bienestar en el orden social en que los individuos ostentan su posición. El "uso público de la razón", por el contrario, supone el interés desinteresado de un deseo singular-universal, que involucra la decisión de un sujeto, la felicidad de dejarse capturar, implicar, por el acontecer de una verdad. La lógica del primero es la de la imposición flexible de la lengua materna, arraigada en la tierra, la cultura y en los lugares identitarios que reparte el contexto; mientras la del segundo es la del lenguaje alfabético, esa violenta y traumática sustracción instituyente que perfora el trayecto de los saberes significativos.

Los estudios superiores universitarios se sustentan en la tensión siempre conflictiva entre lo profesional y lo académico, entre el título profesional y el grado académico. Esta tensión podríamos describirla de la siguiente forma: en lo profesional se trata de la adquisición de un conjunto de saberes y habilidades que promuevan una sólida competencia para resolver 
los problemas del campo disciplinario en cuestión, dispuesto al servicio de la sociedad; en lo académico, por el contrario, se trata más bien, por un lado, de saberes y verdades que permitan pensar los cuadros conceptuales y las cartografías de compromisos y creencias que organizan a las disciplinas para problematizarlas crítica y reflexivamente, y por otro lado, en un segundo momento, se trata de que desde esa problematización podamos decidir afirmativamente intervenciones teórico-prácticas, que esta vez no se subordinan simplemente a los requerimientos del orden social, pues se orientan por ideas que posibiliten, más bien, su verdadera transformación; no el círculo vicioso y paradojal de la innovación y la creatividad requeridas para adaptarse mejor a lo que hay. La dimensión académica es necesariamente transdisciplinar, pues pone las condiciones para que el estudiante tome la requerida distancia de su disciplina, precisamente para poder entrar en relación con ella, con sus presupuestos y gramáticas. Así, si asumimos que la institución universitaria en particular, y la educativa en general, no se reduce a una empresa prestadora de servicios técnicos de calidad para mejor brindar prestaciones profesionales, entonces hay que asumir que la educación se instituye ante todo como instancia de una singular filiación simbólica, al modo de un comienzo sin origen, en la tensión entre lo profesional y lo académico. Lo que ante todo está en juego es la inscripción de una falta, la transferencia de un deseo, como la instancia que permite la erotización del pensamiento, la inscripción de un deseo emancipador, o sea, de una orientación. Aquí la posición del profesor no es la de mero mediador, relator o facilitador de información, que es más bien la figura del fracaso funcional que propicia la psicopedagogía; ceder en el propio deseo (Lacan, 2003), renunciar al acto educativo.

En este sentido, cuando se habla de educación de calidad, pareciera que el problema de cómo asegurarla está ahí para cubrir el problema de la $e d u$ cación. No negamos la importancia de los indicadores y las mediciones, siempre y cuando se repare en que esta dimensión atiende a los factores de organización, de gestión disciplinaria y profesional, que hay que sostener en su conflictiva relación con las instancias académicas. Lo que hemos llamado acto educativo refiere a los efectos subjetivantes e instituyentes que posibiliten el ingreso en un orden simbólico, lo que en términos de la Ilustración se llama formación. Se trata de sostener la torsión de la 'instrucción', la adquisición de habilidades y conocimientos, llevada a cabo por la 'formación' al modo de un cambio de dirección emancipatorio. En esta conjunción se despliega, sin garantía última, todo proceso educativo. En su traducción o reactualización de La República de Platón, Badiou (2013b) señala: 
la educación no es entonces una cuestión de imposición, sino de orientación. Es, diría, una técnica de conversión. Lo único importante es encontrar el recurso más simple y más eficaz para que se opere ese cambio total del Sujeto. No se trata de ningún modo de imponerle la vista: ya la tiene. Pero como está mal dirigida y no se vuelve hacia las realidades adecuadas, hay que reorientarla a toda costa. (p. 281)

La calidad puede ser un expediente que pretenda garantizar las condiciones materiales y objetivas, tales como la confortabilidad de los espacios, las salas de clases, la biblioteca, los estudios de los profesores, etc. Y esto es medible y verificable empíricamente. Pero el acto educativo se juega en una dimensión subjetiva, es decir, en la relación con un Otro por medio de la ley de la palabra. A la "lógica diferencial" y a la "lógica oposicional" entre institución y organización parece escapárseles un punto. El problema no es que la organización haya desplazado a la institución, el problema es que en ese desplazamiento la institución queda, permanece, pero a costa de perder su eficacia simbólica, su dimensión instituyente. Así, no cabe duda que en un contexto de masificación de la educación son necesarios criterios de organización que permitan evaluar las nuevas condiciones, pero para

suprimir los problemas que esta bienvenida masificación genera en la institución, no para suprimir aquello que la define. En este sentido nuestra situación es similar a la que señala Chesterton (2008) en Lo que está mal en el mundo:

Hace un tiempo algunos médicos y otras personas a las que la ley moderna autorizó a dictar normas a sus conciudadanos menos elegantes emitieron una orden que decía que había que cortar el pelo muy corto a las niñas pequeñas.

Me refiero, naturalmente, a aquellas niñas pequeñas cuyos padres fueran pobres (...) En consecuencia, los médicos sugieren suprimir el pelo. No parece habérseles ocurrido suprimir los piojos. Y, sin embargo, eso se podría hacer. (p. 240)

Para concluir, podemos ensayar a partir de lo expuesto un correlato entre los tres momentos del orden simbólico descritos por Badiou (2016) en La verdadera vida, y los tres tipos de Escuela propuestos por el psicoanalista Massimo Recalcati en La hora de clase (2016), p. 1) Simbólico jerárquico - Escuela Edipo: aquí se puso en juego la institución jerárquica del mundo de la tradición, configurado por el ideal de un deseo identitario y un sentido único del que la religión sacó todos sus dividendos; 2) A-simbólico desigualitario - Escuela Narciso: la caída de los ideales trae consigo el des- 
orden de lo Simbólico, lo que da lugar a la facticidad del orden que impone el saber experto, al modo de una organización corporativa de la desigualdad, sustentada en el desánimo maniaco-depresivo del relativismo nihilista posmoderno, que se agota en el deseo anónimo de los procedimientos estandarizados y la credencialización del individuo como empresario del yo; 3) Simbólico igualitario - Escuela (Antígona-) Telémaco: mundo por-venir -a verificar en el presente de las Ideas y las decisiones que inscriben en el sujeto la singularidad de un deseo instituyente. Este correlato se extiende diacrónica y sincrónicamente. En esta medida no se trata de esperar un determinismo histórico que se despliegue como un trayecto y un rumbo garantizados, ni de quedarse a la espera en la inminencia de lo porvenir. Por ello a lo largo de este escrito hemos tratado de componer una trenza entre el sujeto, las verdades y el deseo en su dimensión instituyente, para no quedar entrampados en la mera demanda reivindicativa y en la sola crítica disolvente. Al necesario diagnóstico crítico que dilucida las condiciones de posibilidad de la situación contemporánea de la educación es necesario suplementarlo, como hemos tratado de hacerlo, con esta orientación afirmativa que condensa el aserto del acto educativo.

\section{REFERENCIAS}

Atria, R. y Lemaitre, M. J. (2014). El aseguramiento de la calidad en el escenario cambiante de la educación superior. Revista Estudios Sociales, 122, 9-36.

Badiou, A. (2006). Lógicas de los mundos. El ser y el acontecimiento, 2. Buenos Aires: Manantial.

Badiou, A. (2007a). ¿Se puede pensar la política? Buenos Aires. Nueva Visión. Badiou, A. (2007b). El ser y el acontecimiento. Buenos Aires. Manantial. Badiou, A. (2009). Pequeño manual de inestética. Buenos Aires: Prometeo.

Badiou, A. (2013a). La aventura de la filosofía francesa. Buenos Aires: Eterna Cadencia.

Badiou, A. (2013b). La Republica de Platón. Buenos Aires: Fondo de Cultura Económica.

Badiou, A. (2016). La verdadera vida. Barcelona: Malpaso.

Bellei, C. (2015). El gran experimento. Mercado y privatización de la educación chilena. Santiago: Lom.

Cancino, V. y Schmal, R. (2014). Sistema de Acreditación Universitaria en Chile: ¿Cuánto hemos avanzado? Revista Estudios Pedagógicos, XXI(1), 41-60.

Chaui, M. (2018a). La ideología de la competencia. Barcelona: NED.

Chaui, M. (2018b). Contra la universidad operacional. En W. Thayer, E. Collingwood, M.L. Estupiñán, R. Rodríguez (eds.). La universidad (im)posible (pp. 224-236). Santiago: Ediciones Macul. 
Chesterton, G. K. (2008). Lo que está mal en el mundo. Barcelona: Acantilado.

De Lajonquiére, L. (1999). Infancia e ilusión (Psico)-Pedagógica. Escritos de psicoanálisis y educación. Buenos Aires. Nueva Visión.

Díaz, G. (2016). Calidad. En M. L. Estupiñán. El ABC del neoliberalismo (pp. 183-210). Viña del Mar: Communes.

Espinoza, O. y González, E. (2012). Estado actual del Sistema de aseguramiento de la calidad y el régimen de acreditación en la educación superior en Chile. Revista de la educación superior, XLI(2), 87-109.

Fernández, C. (2009). El plan Bolonia. Madrid: Catarata.

Fernández, C., García, O. y Galindo, E. (2017). Escuela o barbarie. Entre el neoliberalismo salvaje y el delirio de la izquierda. Madrid: Akal.

Harvey, L. \& Green, D. (1993). Defining Quality. Assessment \& Evaluation in Higher Education, 18(1), 9-34, DOI: 10.1080/0260293930180102.

Kant (2004) ¿Qué es la Ilustración? Madrid. Alianza.

Lacan, J. (2003). La ética del psicoanálisis. El seminario 7. Buenos Aires: Paidós.

Lacan, J. (2009a). La transferencia. El seminario 8. Buenos Aires: Paidós.

Lacan, J. (2009b). La relación de objeto. El seminario 4. Buenos Aires: Paidós.

Laclau, E. (2002). Misticismo, retórica y política. Buenos Aires: Fondo de Cultura Económica.

Lemaitre, M. J. y Zapata, G. (2003). Antecedentes, situación actual y perspectivas de la evaluación y acreditación de la educación superior en Chile. Demandas Sociales y Gestión del Conocimiento (pp. 147-184). Santiago: Centro Interuniversitario de Desarrollo CINDA.

Marshall, M.T. (2014). Aseguramiento de la calidad: discursos y observaciones desde el consejo de Rectores. Revista Estudios sociales (Chile), 122, 193-215.

Mayol, A. (2012). No al lucro. De la crisis del modelo a la nueva era política. Santiago: Debate.

Miller, J.-A. (2011). Recorrido de Lacan. Buenos Aires: Manantial.

Mönckeberg, M. O. (2007). El negocio de las universidades en Chile. Santiago: Random House Mondadori.

Mönckeberg, M. O. (2013). Con fines de lucro. La escandalosa historia de las universidades privadas en Chile. Santiago: Debate.

Mouffe, C. (2007). En torno a lo político. Buenos Aires: Fondo de Cultura Económica.

Orellana, V. (ed.) (2018). Entre el mercado gratuito y la educación pública. Dilemas de la educación chilena actual. Santiago: Lom.

Paradeise, C., y Thoening, J-C. (2017). En busca de la calidad perdida. Ciudad de México: Fondo de Cultura Económica.

Pesqueux, Y. (2009). Institución y organización. Revista Cuadernos de administración, 25(41), 9-25.

Readings, B. (2012). La idea de excelencia. En R. Rodríguez y M. Tello. Descampado. Ensayos sobre las contiendas universitarias (pp. 183-210). Santiago: Sangría Editores. 
Recalcati, M. (2016). La hora de clase. Por una erótica de la enseñanza. Barcelona: Anagrama.

Ruiz, C. (2010). De la república al mercado. Ideas educacionales y política en Chile. Santiago: Lom.

Salazar, J. y Leihy, P. (2014). La poética del mejoramiento: ¿Hacia dónde nos han traído las políticas de calidad de la educación superior? Revista Estudios sociales, 122, 125-191.

Schejtman, F. (2013) Ensayos de clínica psicoanalítica nodal. Buenos Aires: Grama.

Vergara, J. y Martin, A. (2016). Pensar la educación. Desde Friedman a Dewey. Santiago: Editorial Universitaria.

Vidal, P. (2017). La educación superior chilena como campo de disputa ideológica. Santiago: Ediciones UAHC. 